SPECIAL ISSUE

\title{
Tele-Mentoring and Monitoring of the National Mental Health Program: A Bird's-Eye View of Initiatives from India
}

\author{
Gopi Gajera', Barikar C. Malathesh', Lakshmi Nirisha P', Channaveerachari Naveen \\ Kumar'*, Narayana Manjunatha', Suchandra H.H.', Sujai Ramachandraiah', Chethan \\ Basavarajappa', Rajendra Gowda K.M. ${ }^{3}$ and Suresh Bada Math'
}

\begin{abstract}
'Telemedicine Centre, Department of Psychiatry, NIMHANS, Bengaluru, Karnataka, India; ${ }^{2}$ Department of Psychiatry, NIMHANS, Bengaluru, Karnataka, India; ${ }^{3}$ Department of Child and Adolescent Psychiatry, NIMHANS, Bengaluru, Karnataka, India
\end{abstract}

Abstract

Objective: To provide a glimpse of various digital programs and modules that are being implemented across the country by the National Institute of Mental Health and Neurosciences (NIMHANS), Bengaluru, India (an institution of national importance under the Ministry of Health and Family Welfare, Government of India; one of its mandates is to develop innovative strategies to improve mental health capacity building as part of the National Mental Health Program, a publicly funded health program to cater to the public health need posed by psychiatric disorders).

Design: The information is presented in a narrative fashion by organizing the activities into three categories of digital training methods: webinar mode, blended mode and hybrid mode.

Results: Cadres ranging from lay-counsellors (volunteers in the community), non-specialist health workers to professionals including medical officers are covered with these initiatives. During the period from August 2016 till December 2020, more than 16 million man hours of training is delivered for more than 35,000 participants from across the country.

Conclusions: These have a tremendous potential to exponentially increase skilled human resources capable of providing quality care to hitherto unserved remote areas of the rural hinterland and ultimately reduce the burgeoning treatment gap. In-depth outcome assessments are the need of the hour.

Keywords: Capacity building; Tele-psychiatry; Tele-mentoring and monitoring; Primary care psychiatry

$\mathrm{T}$ he World Health Organisation (WHO) defines telemedicine as 'The delivery of healthcare services, where distance is a critical factor, by all healthcare professionals using information and communication technologies for the exchange of valid information for diagnosis, treatment and prevention of disease and injuries, research and evaluation and for the continuing education of healthcare providers, all in the interests of advancing the health of individuals and their communities' (1). In March 2020, telemedicine practice guidelines (TPG) were released by MCI and the Government of India following which in May 2020, the Indian Psychiatric Society (IPS) and the Telemedicine Society of India (TSI) in collaboration with the National Institute of Mental Health and Neurosciences (NIMHANS) released the telepsychiatry operational guidelines (TOG) $(2,3)$.
Although technology is being extensively utilized for clinical care in India, emerging trends indicate its growing utility in mental health capacity building (4). This is driven by the obligatory need of reaching out to the burgeoning number of people with psychiatric disorders, amounting to about 150 million (NMHS, 2016; Mental Healthcare Act, of India; 2017) (5). Added to this, the number of skilled human resources are deficient (6). Also, there is significant inequality in their distribution. Lastly, there is a lack/absence of training in psychiatry during undergraduate medical education and internship $(7,8)$. There is inadequate knowledge related to the diagnostic criteria of common mental disorders, lack of awareness, the appropriate questions to ask, and time limitations inherent in a busy primary care clinic setting which lead to significant underdiagnosis of mental health issues (9). 
In order to provide basic mental health services, the Government of India in association with health departments of the state governments, is running the National Mental Health Program (NMHP) that has District Mental Health Program (DMHP) as one of its core components. Providing basic mental health services at the district level is the most important objective of DMHP. Though it is operational in 650 districts (out of the total 725), its implementation is patchy (10-12). The onerous task of equipping the ground level health force in identifying and managing common still remains. The traditional training programs that involve didactic lectures, video demonstrations, PowerPoint presentations in classroom and group format often fail to translate into clinical skills required for early diagnosis and treatment of psychiatric disorders. Principles of adult learning are absent in most of the typical class room teaching (CRT) and follow a top-down approach (13).

To overcome the drawbacks of traditional CRT, the Telemedicine Centre, Department of Psychiatry at NIMHANS, Bengaluru, India, has designed multiple modules for training and empowering primary care health providers as well as under graduate students and post graduate psychiatry students. These programs are digitally driven, based on the principles of adult learning and extended handholding.

\section{Objectives}

In this paper, we review the various telepsychiatry-related capacity building programs, projects, and services undertaken to do tele-mentoring and monitoring under the National Mental Health Program across the country.

\section{Results}

These digital modules can be broadly divided into three major modes as follows (14):

- Webinars: Synchronous interactions in hub and spokes mode.

- Blended mode: Combination of synchronous and asynchronous interaction using e-learning application and video conferencing (only online interaction, without any in-person interaction).

- Hybrid mode: Initial onsite training followed up by online training using both synchronous and asynchronous methods (includes both in-person and online interactions).

\section{Capacity building - webinar mode}

Indians (IPS-South Zone \& NIMHANS Digital Initiative for Academic \& Nurturing Skills)

This is an initiative by NIMHANS in collaboration with the south zone of the Indian Psychiatric Society - South
Zone and was inaugurated on 8 June 2019. This is an online teaching initiative comprising weekly sessions of 1 $\mathrm{h}$ each, through a multipoint videoconference platform. The training focuses on psychiatric subspecialties using didactic classes and case-based learning. Initially, the program had 645 psychiatry residents pursuing MD/DNB/ DPM and 471 practicing psychiatrists got registered for the INDIANS program. At present the sessions are broadcasted live on the YouTube channel of NIMHANS which has more than 2,670 subscribers majority of whom are psychiatrists (15). The views of the YouTube channel have seen a substantial hike in the year 2020 following the COVID pandemic, highlighting the increased utilization of technology-based learning platforms. The number of views in 2019 was 3,347 and in 2020 it was 29,461 views. This online program was present before Covid-19 became popular and was able to increase the number capacity building activities during the lockdown.

\section{Public psychiatry activities}

Various departments/specialty units like adult psychiatry, child and adolescent psychiatry, geriatric psychiatry, addiction psychiatry, forensic psychiatry, clinical psychology, psychiatric social work and integrated medicine have conducted academic training in synchronous mode in the form of webinars. Beneficiaries range from the general population, care givers of the mentally ill, lay counsellors, college students, school/college teachers, accredited social health activist workers, yoga trainers, social workers, clinical psychologists, nurses, judges, lawyers, police personals, doctors, obstetricians, neurosurgeons, anesthesiologists and psychiatrists. Typically, the duration of such sessions is $1 \mathrm{~h}$. Figure 1 demonstrates the sessions that were held at a telemedicine center from 2017 till the date shown. We can see the drastic hike in video conferences conducted in 2020 .

Various virtual activities were conducted by different departments for awareness and training in psychosocial wellbeing during Covid-19. A total of 39 programs were conducted addressing more than 30,000 national and international audience.

\section{Capacity building: blended mode}

\section{NIMHANS digital academy (NDA)}

NDA was inaugurated in June 2018 with the vision of exponentially increasing human resources to cater to mental health ailments by leveraging technology (16). It offers the following two types of digital courses incorporating both synchronous and asynchronous modes of learning.

Diploma in community mental health: This blended diploma (of 3 months duration, spanning 3 months) provides a basic understanding of the common mental health conditions and enables one to screen, identify, treat and to 


\section{Sessions at Telemedicine Centre}

600

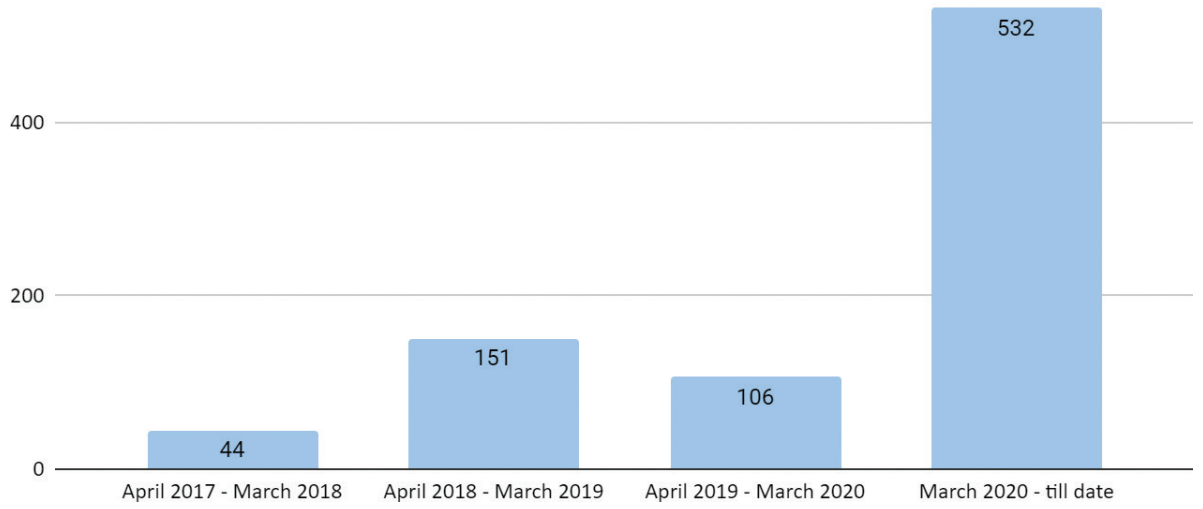

Figure 1. Number of sessions held at NIMHANS-telemedicine center from 2017 till the date shown.

Table 1. List of certificate courses with duration target groups

\begin{tabular}{|c|c|c|}
\hline Name & Hours/weeks & Target group \\
\hline $\begin{array}{l}\text { Basics of addiction management } \\
\text { CCBoAM for nurses and counsellors }\end{array}$ & $20 \mathrm{~h} / \mathrm{I} 2$ weeks & Nurses and counsellors \\
\hline Suicide prevention gatekeeper training : Chhattisgarh & $24 \mathrm{~h} / \mathrm{I} 2$ weeks & Counsellors from Chhattisgarh \\
\hline Psychotropic medication:What a doctor should know & $24 \mathrm{~h} / \mathrm{I} 2$ weeks & Doctors \\
\hline Neurodevelopmental & $30 \mathrm{~h} / 20$ weeks & Doctors \\
\hline Perinatal mental health & $30 \mathrm{~h} / \mathrm{I} 2 \mathrm{wks}$ & $\begin{array}{l}\text { Doctors } \\
\text { Counsellors }\end{array}$ \\
\hline Psychosocial effect of Covid-19 & $30 \mathrm{~h} / 12 \mathrm{wks}$ & Counsellors \\
\hline Certificate course in basics of addiction management & $70 \mathrm{~h} / 24$ week & $\begin{array}{l}\text { Doctors } \\
\text { Counsellors } \\
\text { Nurses }\end{array}$ \\
\hline Basics of alcohol addiction management (BoAAM) ECHO for GPs: Odisha & $40 \mathrm{~h} / \mathrm{I} 2$ weeks & Doctors \\
\hline Dementia & $40 \mathrm{~h} / 12$ week & Doctors \\
\hline
\end{tabular}

BoAAM: basics of alcohol addiction management; CCBoAM: certificate course in Basics of Addiction Management; ECHO: Extension for Community Healthcare Outcomes.

refer. It includes six virtual sessions fortnightly and selfpaced learning material and two E-consults and monthly reporting of cases seen and cared for. The Diploma in Community Mental Health (DCMH) is designed for doctors $(30 \mathrm{~h})$, nurses $(25 \mathrm{~h})$, psychologists $(25 \mathrm{~h})$, and social workers $(25 \mathrm{~h})$. At the end of the training, eligible participants are awarded with DCMH. Till date, a total of 1,734 primary care doctors (PCDs), 786 psychologists, 378 social workers and 614 nurses have enrolled for DCMH from July 2018 to date.

Certificate courses: These courses are designed as per the request from various agencies and state governments. These courses too have a blended approach with virtual face-to-face and self-paced learning over fixed duration. Typically, the sessions will be conducted twice in a month. E-learning material will be shared every week. At the end of the training, eligible participants will be awarded with a participation certificate. Table 1 describes various types of certificate courses with the hours of training in each of them and the number of participants enrolled. A total of 628 participants have enrolled for these courses (Table 1).

\section{Capacity building: hybrid mode}

This hybrid mode of training is primarily being conducted for PCDs across different states under the broader umbrella of 'primary care psychiatry'. The training programs consist of different modules used in different combinations. First, we outline the modules followed by details about specific programs.

Curriculum - clinical schedule of primary care psychiatry (CSP) CSP is an all-in-one integrated, validated tool for PCDs for highly prevalent six psychiatric disorders at primary care. These six disorders are tobacco addiction, alcohol 
(harmful and addiction), psychotic, depressive, anxiety (panic and generalized anxiety disorders), and somatization disorders (abbreviated as as TAP DAS). CSP consists of the screening questions, diagnostic guidelines, and management guidelines (pharmacotherapy, prescription templet, side-effects, follow-up guideline, \& referral guidelines). CSP was tested on 180 patients by PCDs and further interviewed by psychiatrists, wherein agreement was found between diagnoses made by PCDs and psychiatrists for $142(78.9 \%)$ patients with a Cohen's kappa $(K)=0.57$. The sensitivity was $91.1 \%$ and specificity was $68.3 \%$ with interrater reliability of $K=0.7$. CSP is an effective tool for screening, diagnosing and treating the psychiatric patients at the primary healthcare (17).

\section{Training modules for primary care psychiatry (I 3)}

\section{Classical onsite module}

Onsite training is nothing but the classical classroom teaching, where a general orientation towards psychiatric disorders is imparted. Additionally, participants are also given an outline of the entire programme, various digital modules and in-person observation of psychiatry consultations. Typically, it forms a minor part of the whole program.

\section{Digital modules}

These are four in number and are described in the following paragraphs.

Tele- on consultation training (Tele-Oct): It based on the hub (tele-psychiatrists at NIMHANS) - and - spoke (PCD) model. OCT is an on-job/hand-holding training conducted in live, real-time clinical scenarios while PCDs are providing consultations to their general patients. The goal of OCT is to maximize the quality of the general practice of PCDs with the provision of providing standard psychiatric care to patients with psychiatric disorders. It incorporates the principles of 'real patients, real consultations, but with new clinical practice acumen'. It causes minimal disruption to their clinical work. It incorporates principles of adult learning, a bottom-up approach wherein each and every clinical skill is taken care separately from entry-to-exit of patients. The curriculum used in training is CSP. Typically, there are one-to-one sessions and each session lasts for about $2-3 \mathrm{~h}$, wherein 10-15 consecutive patients are seen. In the first session, tele-psychiatrists will demonstrate interviewing skills and teach different guidelines from CSP. During the second session, PCDs will be asked to see the patients of his/her outpatient department and screen them for psychiatric illness using the screener provided in CSP; while this is ongoing, the tele-psychiatrist will be observing the PCD and will guide him/her in using the diagnostic guidelines and management guidelines. Typically, in the second session the tele-psychiatrist will take the lead in assessing and diagnosing the patient. During the third and fourth sessions, PCDs will take the lead and the psychiatrist will observe and intervene only if necessary. Typically, the frequency of the sessions are at 1st week, 3rd week, and 7 th week after the initial training. The Tele-OCT has dual outcomes: one is training PCDs and the other is providing consultation from collaborative care $(13,17)$

Virtual classroom (VCR): It is based on the hub (telepsychiatrists at NIMHANS)-and-spoke (PCD) model and the principle of peer learning. It includes interactive sessions in the form of seminars, case discussions, and expert lectures. The seminar on selected topics will be presented by the trainee to their fellow participants in the presence of a tele-psychiatrist. Typically, such a session would be conducted weekly and the total duration of the session would be around $1 \mathrm{~h}$ (11).

Collaborative video consultation (CVC): this module is based on the practice-based learning using one-to-one consultation with the patient and PCD. Live and real-time video-based assistance will be provided by a telepsychiatrist on all working days for discussion of selected cases by PCDs. PCDs chose cases for discussion depending upon the difficulty level of the case, and in collaboration with tele-psychiatrists they decide the best management plan for selected patients. The tele-psychiatrist uses the casebased learning approach and video demonstration to help PCD gain the skill of interviewing the psychiatric patient. Typically, such a session would last for 15-20 min.

Virtual case conference (VCC): It is based on the hub (telepsychiatrists at NIMHANS)-and-spoke (PCD) model and the principle of peer learning. It includes interactive sessions in the form of virtual live case discussion, on prefixed date and time, with tele-psychiatrists while the fellow PCDs are connected through different spokes and observe the case discussion and live patient interview to obtain clinical skills.

\section{Capacity building projects with states - using telemedicine}

\section{Mandya pilot project}

The pilot for training PCDs began in Mandya District of Karnataka State from August 2016 to August 2018. CSP was used as the curriculum and digital modules included Tele-OCT and CVC. It also included 3 days of classical onsite training for 133 PCDs. Out of them, 71 participants completed their online training. Each doctor got four OCT sessions with a gap of 1 month each. A total 113 Tele-OCT sessions were successfully conducted. A total of 823 patients were seen during this training period.

District Mental Health Plan (DMHP) Uttarakhand (UK)

After gaining experience from the Mandya Project, NIMHANS collaborated with the Uttarakhand State 
Government to train PCDs. Over a period of 2 years, 21 PCDs were trained and awarded 'Diploma in Primary Care Psychiatry', a 1-year diploma that is a parttime course that can be earned without hampering their routine service. DPCP comprises a total five modules. First 1-3 days comprise the onsite orientation module, 10-14 days comprise the onsite basic module, advanced module (2-4 Tele-OCT), weekly VCR sessions and continuous hand holding via CVC (13). A total of 2,182 patients were consulted through various digital modules through this training. Each PCD underwent a minimum of $108 \mathrm{~h}$ of training. A total of 2,182 patients were consulted through various digital modules through this training.

\section{The Karnataka Telemedicine Mentoring and Monitoring Program} (KTM)

The KTM program is a collaborative program between NIMHANS and the government of Karnataka, which was conducted from January 2019 to October 2020. This model had a four tier hub and spoke model, consisting of 'hub', 'micro hub', 'mini-hub', and 'spokes'. 'Hub' was represented by a KTM trained psychiatrist who was present at NIMHANS. 'Micro-hub' comprised the psychiatrist in DMHP, who was at the district headquarters, 'Mini hub' was the PCD at Primary Health Centres (PHC) and 'spokes' were the patients. Psychiatrists employed under the DMHP were trained in conducting (TOT Model) Tele-OCT and CVC. The above trained psychiatrist further handheld the PCDs at the PHC and provided training in identifying and managing psychiatric illnesses. A total of 33 DMHP psychiatrists were trained and they in-turn trained 436 PCDs across the state via Tele-OCT and CVCs. The training was held under the monitoring of telepsychiatrists from NIMHANS. This was a unique program which imparted training to the trainer (18). Also, a randomized controlled trial was planned to evaluate patient-level outcomes of the KTM program in comparison to the traditional training program, Unfortunately, the Covid-19 pandemic struck and precluded the team to venture into the field. This program received the Digital Innovation award under the public health category from the Government of India.

\section{Chhattisgarh Community Mental Healthcare Telementoring Program (CHaMP)}

The Chhattisgarh community mental healthcare telementoring program (CHaMP) is a training program conducted in collaboration with the government of Chhattisgarh from June 2019 till date. The aim of this program is to train all PCDs of Chhattisgarh $(n=2,180)$ in screening, identifying, and treating common mental disorders. CSP is used as the curriculum and three different digital modules are used, namely VCR, VCC, and CVC. It is a 8 weeks program, with 2 days of onsite training and weekly $2 \mathrm{~h}$ online session on CSP with VCC. A total of 905 participants have received the training so far. At the end of the training eligible participants would receive a certificate titled 'certificate course in primary care psychiatry'. All the trained PCDs are advised to share a monthly report of the number of psychiatric consultations conducted. More than 20,000 psychiatric consultations are reported by them till November 2020.

\section{Primary Care Psychiatry Project for Bihar}

NIMHANS in collaboration with the government of Bihar has launched a 1-year diploma course in Primary Care Psychiatry to train PCDs from February 2020 till date. Despite the COVID-19 pandemic, 22 doctors took up the training and were able to engage in tele-OCT sessions keeping adequate social distancing norms and safety measures. All the trained PCD are advised to share a monthly report of the number of psychiatric consultations conducted. More than 2,800 psychiatric consultations are reported by them, till Nov 2020.

Table 2 describes the details of the training hours in onsite and online mode and the number of patients consulted through video consultation and tele-OCT for all the government liaised projects.

\section{Lay counsellors training}

NIMHANS in association with Apthasalaha Kendra, a non-governmental organisation (NGO) started training the lay-counsellors (members of the civil society interested in providing psychological first aid to the distressed individuals). This initiative has now been formalized into

Table 2. Online training delivered from hub side for various government-aided projects

\begin{tabular}{lcccccccc}
\hline & No. of participants & Total onsite & Total online duration & VCR & CVC & Tele-OCT & VCC & Total patients \\
\hline Mandya Project & $7 \mathrm{I}$ & $18 \mathrm{~h}$ & $555 \mathrm{~h}$ & - & - & $\mathrm{II}(555 \mathrm{~h})$ & - & 824 \\
Uttarakhand & $2 \mathrm{I}$ & $108 \mathrm{~h}$ & $860 \mathrm{~h}$ & $79(79 \mathrm{~h})$ & $60 \mathrm{I}(124 \mathrm{~h})$ & $104(180 \mathrm{~h})$ & - & 2,182 \\
KTM & 469 & $6 \mathrm{~h}$ & $1,062 \mathrm{~h}$ & $\mathrm{I}(2 \mathrm{~h})$ & $27(7 \mathrm{~h})$ & $598(\mathrm{I}, 054)$ & - & 4,484 \\
CHaMP & 905 & $108 \mathrm{~h}$ & $627 \mathrm{~h}$ & $273(273 \mathrm{~h})$ & $32 \mathrm{I}(80)$ & - & $273(273 \mathrm{~h})$ & 577 \\
Bihar & 22 & $42 \mathrm{~h}$ & $98 \mathrm{~h}$ & $32(52 \mathrm{~h})$ & $42(10 \mathrm{~h})$ & $31(36 \mathrm{~h})$ & - & 436 \\
\hline
\end{tabular}

CHaMP: Chhattisgarh Community Mental Healthcare Telementoring Program; CVC: Collaborative Video Consultation; KTM: Karnataka Telemedicine Mentoring and Monitoring; VCR: virtual classroom; VCC: Virtual Case Conference, h: hours. 
a 'certificate course in lay-counselling'. This course consists of $36 \mathrm{~h}$ of online face-to-face training and $16 \mathrm{~h}$ of onsite observation. The latter incorporates visits to psychiatry outpatient clinics to observe and learn clinical interactions between patients and experts.

It is a blended learning approach including both synchronous and asynchronous modules consisting of observational learning, self-paced learning and discussion with roleplay. In the asynchronous mode, counsellors (spoke) go through e-learning assignments allotted to them via the phone-based applications, which would be evaluated, and a feedback will be given at a later point. Synchronous mode involves didactic lectures, case presentations (by counsellors) and discussion with NIMHANS team (spoke) for a duration of $2 \mathrm{~h}$.

\section{Digital portal (e-Manas)}

Karnataka Mental Health Care Management System (KMHMS) (19)

The KMHMS or e-Manas ('Manas' a Sanskrit word means 'mind') online portal is conceptualized and developed to help manage, monitor, and implement the provisions contained in the Mental Healthcare Act (MHCA 2017). People with mental illness (PwMI), their caregivers, Mental Health Establishments (MHEs), Mental Health Professionals (MHPs), and regulatory bodies like Karnataka State Mental Health Authority (KSMHA) and District Mental Health Review Boards (MHRBs) are brought together under a single online web-based solution to provide integrated and seamless mental health care service delivery.

Inputs about medical and legal requirements as prescribed in MHCA 2017 and continuous assistance to website development was provided by the team from NIMHANS, Bengaluru. Technical aspects, development, and testing of the web application were undertaken by the team from E-Health Research Centre, International Institute of Information Technology (IIIT-B) Bengaluru. Portals were developed for registering MHEs and MHPs, for documenting patient care, for patients to upload valid Advance Directives (AD) and raise grievances against specific MHE/MHP, for authorities to approve the registrations and regulate the service delivery according to the legislation. The portal is also integrated with Ayushman Bharat-Arogya Karnataka (AB-ARK) Database, Arogya Sahayavani, and emergency ambulance services. The website has been launched and handed over to the government of Karnataka on July 2020.

\section{Research}

Implementation research evaluating outcomes of digital training initiatives in India

One of the important components of these digital training initiatives is the outcome assessments. These will provide the utility, acceptability, scalability, and shed light on barriers to upscale them. Many of the above training initiatives have hand in hand looked into the outcomes and it is heartening to note a number of positive outcomes such as: (a) Feasibility of connecting an academic hub to remote districts of states in the country (20); (b) utility of central venous cannula (CVCs) in managing complex opioid dependence cases by a PCD (21); (c) establishing validity and reliability of 'Clinical Schedules for Primary care Psychiatry' (17); (d) acceptable level of retention of skills and utility of these skills in managing patients (21). Further, a randomized controlled trial is underway to prospectively compare the outcomes of the traditional training module and digitally driven training module on a number of patient related outcomes (22). In addition to opening newer avenues to overcome the traditional barriers, use of technology are associated with a few challenges as well, particularly when we talk about the field level workers (23). Clearly, more data is needed in this area.

\section{Conclusions}

This article summarizes the relatively new and emerging arena of capacity building initiatives undertaken to mentor, monitor, and implement the National Mental Health Program in India. As can be seen, these have a tremendous potential to exponentially increase skilled human resources capable of providing quality care to hitherto unserved remote areas of the rural hinterland and ultimately

Table 3. Hours of training received by each spoke for various government-aided projects

\begin{tabular}{lccccccc}
\hline & Total Onsite & Total online duration & VCR & CVC & Tele-OCT & VCC & Total duration of course \\
\hline Mandya Project & $18 \mathrm{~h}$ & $12 \mathrm{~h}$ & - & - & $4(\mathrm{I} 2 \mathrm{~h})$ & - & 2 months \\
UK & $54 \mathrm{~h}$ & $64 \mathrm{~h}$ & $40(40 \mathrm{~h})$ & $25(6 \mathrm{~h})$ & $6(18 \mathrm{~h})$ & - & 12 months \\
KTM (TOT mode) & $6 \mathrm{~h}$ & $13 \mathrm{~h}$ & - & $10-15(4 \mathrm{~h})$ & $3(9 \mathrm{~h})$ & - & 2 months \\
CHaMP & $12 \mathrm{~h}$ & $13 \mathrm{~h}$ & $6(6 \mathrm{~h})$ & $2(\mathrm{lh})$ & - & $6(6 \mathrm{~h})$ & 2 months \\
Bihar & $42 \mathrm{~h}$ & $76 \mathrm{~h}$ & $52(52 \mathrm{~h})$ & $25(6 \mathrm{~h})$ & $6(18 \mathrm{~h})$ & - & 12 months \\
\hline
\end{tabular}

CHaMP: Chhattisgarh Community Mental Healthcare Telementoring Program; CVC: Collaborative Video Consultation; KTM: Karnataka Telemedicine Mentoring and Monitoring; VCR: virtual classroom; VCC: Virtual Case Conference, h:hours. 
reduce the burgeoning treatment gap. These new technology driven training modules have conceptually been designed to overcome drawbacks of conventional training methods. Also, technology enables better penetration and reach to cover the last trainee in the loop, provided the existent barriers are effectively crossed. Also, release of TPG and TOG have removed major barriers in accessing healthcare and helped to reach the unreached. These conducive policy initiatives have come at an opportune time, expedited by the COVID 19 pandemic. With the help of an effective tool like CSP, primary care psychiatry can be taught with ease to PCDs and they can be equipped in the best clinical practices. The digital modules like Tele-OCT, CVC, VCC, and VCR have potential for high translational quotients with continuity of their clinical practice. These modules can be flexibly tweaked to suit the varied needs of PCDs or states depending on the resources and time they have. Also, these initiatives can easily be adopted to reach out to other ground level health workers (the field level workers). A case in point here is the 'mid-level health providers' of the Health and Wellness Centres, that are coming up throughout the country to cater to the comprehensive primary healthcare under the AYUSHMAN BHARAT health scheme. The former initiative is a potential gamechanger as far as public mental health is concerned provided, technology is appropriately leveraged to train and mentor the cadre. Another cadre of field level workers are the Accredited Social Health Activists (ASHAs). However, a caveat needs to be noted. More rigorous prospective and comparative evaluations are the need of the hour to better define the pros and cons of these digitally driven training initiatives. Future research should examine the sustainability and barriers to reach the last needy person. Also, patient level outcomes including symptom severity, disability, functional status, quality of life, and cost-effectiveness need to be studied. To conclude, telepsychiatry is not just a virtual clinical service but it can also be used to build mental health capacity with the potential to reach the most in need.

\section{Authors' Contributions}

We confirm this manuscript has not been published elsewhere, no parts are plagiarized in any form or degree, and is not under consideration by another journal. All authors approved the manuscript and agree with its submission to Telehealth and Medicine Today.

\section{Acknowledgements}

We thank all the staff of the Telemedicine Centre, NIMHANS for helping us in coordinating and tabulating the information. The authors would also acknowledge all the State Governments and NIMHANS administration for all the support extended to these initiatives.

\section{Funding Statement}

The projects discussed above are funded by various state governments and private institute/organizations. INDIANS was funded by the Indian Psychiatric Society IPS-SZ; NDA courses by the State Govt as mentioned in Table 1; DMHP UK by the government of Uttarakhand; KTM by government of Karnataka, ChaMP by the government of Chhattisgarh; PCPP by the government of Bihar; and KMHMS by the government of Karnataka. The funders had no role in study design, data collection and analysis, decision to publish, or preparation of the manuscript.

\section{References}

1. Dasgupta A, Deb S. Telemedicine: a new horizon in public health in India. Indian J Community Med 2008 Jan; 33(1): 3-8. doi: 10.4103/0970-0218.39234

2. Board of Governors, In supersession of the Medical Council of India. Telemedicine practice guidelines. GOI; 2020. Available from: http://egazette.nic.in/WriteReadData/2020/219374.pdf [cited 8 August 2020].

3. Math SB, Manjunatha N, Kumar CN, Basavarajappa C, Gangadhar BN. Telepsychiatry operational guidelines - 2020. Bengaluru: NIMHANS; 2020.

4. Ibrahim FA, Pahuja E, Dinakaran D, Manjunatha N, Kumar $\mathrm{CN}$, Math SB. The future of telepsychiatry in India. Indian J Psychol Med 2020 Oct 1; 42(5_suppl): 112S-17S. doi: $10.1177 / 0253717620959255$

5. Gururaj G, Varghese M, Benegal V, Rao GN, Pathak K, Singh LK, et al. National mental health survey of India, 2015-16. National Institute of Mental Health and Neuro Sciences, NIMHANS; 2016. Available from: http://indianmhs.nimhans.ac.in/ Docs/Report2.pdf [cited 29 August 2020].

6. Garg K, Kumar CN, Chandra PS. Number of psychiatrists in India: baby steps forward, but a long way to go. Indian J Psychiatry 2019; 61(1): 104-5.

7. Gupta R, Narang RL. Psychiatric training and its practice: a survey of 86 practitioners. Indian J Psychiatry 1987 Oct; 29(4): 349-52.

8. Badrakalimuthu VR, Rangaswamy SV. Mental health practice in private primary care in rural India: a survey of practitioners. World Psychiatry 2009 Jun; 8(2): 124-5. doi: 10.1002/j.20515545.2009.tb00229.x

9. Avasthi A, Varma SC, Kulhara P, Nehra R, Grover S, Sharma S. Diagnosis of common mental disorders by using PRIME-MD Patient Health Questionnaire. Indian J Med Res 2008 Feb; 127(2): 159-64.

10. Identification of psychiatric disorders by rural grass-root health workers: case series \& implications for the National Mental Health Program of India | SpringerLink. Available from: https:// link.springer.com/article/10.1007/s11126-020-09807-5 [cited 23 December 2020].

11. Manjunatha N, Singh G. Manochaitanya: integrating mental health into primary health care. Lancet 2016 Feb 13; 387(10019): 647-8. doi: 10.1016/S0140-6736(16)00286-5

12. Manjunatha N, Singh G, Chaturvedi SK. Manochaitanya programme for better utilization of primary health centres. Indian J Med Res 2017 Feb; 145(2): 163-5.

13. Manjunatha N, Kumar C, Math S, Thirthalli J. Designing and implementing an innovative digitally driven primary care 
psychiatry program in India. Indian J Psychiatry 2018; 60(2): 236. doi: 10.4103/psychiatry.IndianJPsychiatry_214_18

14. Malathesh BC, Ramchandraih S, Basavarappa C, Manjunatha N. The Future of community psychiatry: The role of Telemedicine. In: Moirangthem S, Damodaran D, Manjunatha N, Kumar CN, Math SB, editors. Commuity Psychiatry During Pandemic. 2nd ed. Bengaluru: NIMHANS Publication 182; 2020, pp. 175-85.

15. NIMHANS Indians - YouTube. Available from: https://www. youtube.com/c/NIMHANSINDIANS [cited 25 December 2020].

16. Arora S, Kalishman S, Thornton K, Komaromy M, Katzman J, Struminger B, et al. Project ECHO (Project Extension for Community Healthcare Outcomes): a national and global model for continuing professional development. J Contin Educ Health Prof 2016 Jul; 36: S48. doi: 10.1097/CEH.0000000000000097

17. Kulkarni K, Adarsha AM, Parthasarathy R, Philip M, Shashidhara HN, Vinay B, et al. Concurrent validity and interrater reliability of the 'clinical schedules for primary care psychiatry’. J Neurosci Rural Pract 2019 Jul; 10(3): 483-8. doi: 10.1055/s-0039-1697878

18. Manjunatha N, Kumar CN, Math SB, Thirthalli J, Parthasarathy R, Bhaskarapillai B. Karnataka telepsychiatric mentoring and monitoring (KTM) project. National Institute of Mental Health and Neuro Sciences, NIMHANS; 2019. Available from: https:// www.researchgate.net/project/Karnataka-Telepsychiatric-Mentoring-and-Monitoring-KTM-Project [cited 4 October 2020].
19. Math SB, Manjunatha N, Kumar CN, Dinakaran D, Gowda GS, Rao GN, et al. Mental Healthcare Management System (e-MANAS) to implement India's mental healthcare act, 2017: Methodological design, components, and its implications. Asian J Psychiatr. 2021;57: 102391. doi: 10.1016/j.ajp.2020.102391. Epub 2020 Aug 27. PMID: 33187888.

20. Sagi MR, Aurobind G, Chand P, Ashfak A, Karthick C, Kubenthiran $\mathrm{N}$, et al. Innovative telementoring for addiction management for remote primary care physicians: a feasibility study. Indian J Psychiatr 2018 Oct 1; 60(4): 461.

21. Pahuja E, Kumar TS, Uzzafar F, Manjunatha N, Kumar CN, Gupta R, et al. An impact of a digitally driven primary care psychiatry program on the integration of psychiatric care in the general practice of primary care doctors. Indian $\mathrm{J}$ Psychiatr. 2020 Nov 1; 62(6): 690.

22. Kumar CN, Chand PK, Manjunatha N, Math SB, Shashidhara $\mathrm{HN}$, Basavaraju V, et al. Impact evaluation of VKN-NIMHANS-ECHO model of capacity building for mental health and addiction: methodology of two randomized controlled trials. Indian J Psychol Med 2020 Dec 7; 42(6): 0253717620969066. doi: $10.1177 / 0253717620969066$

23. Malathesh BC, Ibrahim FA, Nirisha PL, Kumar CN, Chand PK, Manjunatha N, et al. Embracing technology for capacity building in mental health: new path, newer challenges. Psychiatr Q [Internet]. 2020; 1-8. doi: 10.1007/s11126-020-09859-7 